\title{
Expression of human DNA mismatch-repair protein, hMSH2, in patients with oral lichen planus
}

\author{
HAO-BO LI ${ }^{1}$, YING-HUAI ZHANG ${ }^{1}$, HUI-ZHEN CHEN ${ }^{1}$ and YONG CHEN ${ }^{2}$ \\ ${ }^{1}$ Oral Medicine Department, Second Hospital of Hebei Medical University, Shijiazhuang, Hebei 050000; \\ ${ }^{2}$ Oral Surgery Department, Central Hospital of Cangzhou, Cangzhou, Hebei 061000, P.R. China
}

Received April 5, 2014; Accepted October 6, 2014

DOI: $10.3892 /$ etm.2014.2053

\begin{abstract}
MSH2 is one of the human DNA mismatch repair genes that plays an important role in reducing mutations and maintaining genomic stability. The aim of the present study was to detect the expression and significance of hMSH2 protein in patients with oral lichen planus (OLP). The expression levels of hMSH2 in the OLP group $(n=51)$ and control group with normal oral mucosa $(\mathrm{NM} ; \mathrm{n}=40)$ were detected using an immunohistochemical method and subsequently assessed. The positive rate of $\mathrm{hMSH} 2$ expression in the OLP group was $52.94 \%$, while the rate was $80 \%$ in the control group, exhibiting a statistically significant difference $\left(\chi^{2}=7.1993 ; \mathrm{P}<0.05\right)$. However, the expression of hMSH2 in the OLP tissues was not shown to significantly correlate with the patient gender, age and type of OLP $(\mathrm{P}>0.05)$. In conclusion, the protein expression levels of hMSH2 in the OLP tissues were significantly reduced as compared with that in the NM tissues, indicating that hMSH2 plays a role in the development of OLP. Therefore, hMSH2 may be used as a biomarker for evaluating the cancer risk of patients with OLP.
\end{abstract}

\section{Introduction}

Mismatch repair (MMR) genes provide repair mechanisms for gene replication $(1,2)$. These genes are not oncogenes or tumor suppressor genes, but an additional type of cancer-related gene. Recently, MMR genes have become increasingly studied to investigate the molecular mechanisms underlying the onset of cancer. The main function of MMR is to identify and repair the mismatched bases in base-specific DNA replication, in order to ensure the high-fidelity of DNA replication, control the occurrence of mutations and maintain the genomic stability (3). Currently, there are nine genes involved in MMR

Correspondence to: Dr Ying-Huai Zhang, Oral Medicine Department, Second Hospital of Hebei Medical University, 215 Heping Road, Xinhua, Shijiazhuang, Hebei 050000, P.R. China E-mail: yinghuaizhang@126.com

Key words: oral lichen planus, mismatch repair genes, hMSH2, immunohistochemistry that encode a variety of MMR proteins, which specifically recognize and repair the mismatched bases $(4,5)$. Mutations in any gene of this system can cause the dysfunction of MMR and result in genetic instability, presenting as replication errors or microsatellite instability, consequently leading to cancer. hMSH2 was the first human MMR gene to be isolated, whose mutations are the highest among the nine MMR genes (6). hMSH2 has been shown to recognize mismatched bases (7), and play an important role in reducing the number of mutations and maintaining the stability of genes.

Oral lichen planus (OLP) is a common non-infectious oral disease, with an incidence rate of $1-2 \%$ in the adult Swedish population (8). The disease is more common among individuals aged $>40$ years, particularly in females (9). The pathogenesis of OLP is multifactorial, and may include autoimmunity, chronic local irritation, mental factors, bacterial or viral infections and other factors $(10,11)$. However, the exact mechanism is unknown. Although OLP is a benign disease, it does have the possibility of cancerization (12). Thus, OLP has been classified as a potentially malignant lesion by the World Health Organization (13). The incidence rate of OLP cancerization varies among different epidemiological surveys, ranging between 0.4 and $5.6 \%$, and commonly between 1 and $2 \%(14,15)$. Thus, the cancer risk, influencing factors and cancerization mechanisms of OLP have attracted increasing attention.

In recent years, the single nucleotide database of hMSH2 has been established $(16,17)$, which has resulted in increased research into the role of the hMSH2 gene in carcinogenesis. The expression of hMSH2 has been reported to change in oral squamous cell carcinoma (18); however, the expression of hMSH2 in OLP and its role in carcinogenesis has yet to be reported.

The aim of the present study was to investigate the role of hMSH2 in the pathogenesis and carcinogenesis of OLP by detecting the expression of hMSH2 in OLP tissues using immunohistochemistry. The results may provide novel ideas for accurately predicting the cancer risk of OLP and developing personalized treatment.

\section{Materials and methods}

Tissue samples. Formalin-fixed, paraffin-embedded, human OLP specimens were obtained from the Pathology Department 
at the Second Affiliated Hospital of Hebei Medical University (Shijiazhuang, China). The specimens included 24 cases of reticular type OLP and 27 cases of erosive/atrophy type OLP. The 51 patients comprising the OLP group included 22 males and 29 females, aged between 36 and 72 years (mean age, 53.54 years). Tissue samples were obtained during surgery, and none of the patients had received any treatment prior to surgery.

In total, 40 cases of normal oral mucosa (NM), obtained from the pericyst during cosmetics surgery of the alveolar bone, were used as a control group (NM group). The group included 22 males and 18 females, aged between 11 and 68 years (mean age, 31.30 years). The study was conducted in accordance with the Declaration of Helsinki and with approval from the Ethics Committee of Hebei Medical University. Written informed consent was obtained from all the participants.

Immunohistochemical analysis. All immunohistochemical analyses were performed using horseradish peroxidase-labeled streptavidin. Tissue sections measuring $4-5 \mu \mathrm{m}$ were mounted on Poly-L-lysine-coated slides (Sigma-Aldrich, St. Louis, MO, USA). Following deparaffinization using a standard protocol, sections were treated with $3 \% \mathrm{H}_{2} \mathrm{O}_{2}$ in methanol for $25 \mathrm{~min}$ to block the endogenous peroxidase activity. The slides were subsequently microwaved in citrate buffer (pH 6.0) for $15 \mathrm{~min}$ at $92-98^{\circ} \mathrm{C}$, and cooled to room temperature. After blocking with normal goat serum (Invitrogen Life Technologies, Carlsbad, CA, USA) for $30 \mathrm{~min}$ at $37^{\circ} \mathrm{C}$, the slides were incubated with a polyclonal rabbit anti-human hMSH2 antibody [diluted 1:75 in phosphate-buffered saline (PBS); SC-22771; Santa Cruz Biotechnology, Dalla, TX, USA] overnight at $4^{\circ} \mathrm{C}$ in a high-humidity chamber. Oral squamous cell carcinoma (OSCC) tissue with known hMSH2 expression served as a positive control. For the negative control, PBS was used instead of a primary antibody. Subsequent steps were performed using an SP kit (Hebei Bohai Biology Co., Ltd.), according to the manufacturer's instructions. Immunoreactivity was visualized using a solution of diaminobenzidine as the chromogen, and the nuclei were counterstained with hematoxylin.

Assessment of staining. All the slides were interpreted by an experienced pathologist, according to the standards reported by Lo Muzio et al (18). Positive staining of hMSH2 was scored in the nucleus and in the plasma with brown particles. At least five randomly selected high-power fields (magnification, $\mathrm{x} 400$ ) were examined. The proportion of immunoreactive cells in 100 cells per field was calculated, in which $<5 \%$ was regarded to show negative expression (-), while $\geq 5 \%$ was considered to indicate positive expression (+).

Statistical analysis. Statistical analysis was performed using the SPSS 17.0 statistical software program (SPSS, Inc., Chicago, IL, USA). The $\chi^{2}$ or Fisher's exact tests were used to compare the differences between groups. Correlation analysis between the expression of hMSH2 and the clinical characteristics was performed using the $\chi^{2}$ test. For all analyses, $\mathrm{P}<0.05$ was considered to indicate a statistically significant difference.
Table I. Expression of hMSH2 in patients with NM and OLP.

\begin{tabular}{lcccc}
\hline & & \multicolumn{2}{c}{$\mathrm{hMSH} 2(\mathrm{n})$} \\
\cline { 3 - 4 } Groups & Cases (n) & + & - & Positive rate (\%) \\
\hline NM & 40 & 32 & 8 & 80.0 \\
OLP & 51 & 27 & 24 & $52.94^{\mathrm{a}}$ \\
\hline
\end{tabular}

${ }^{\mathrm{a}} \mathrm{P}<0.05$, vs. NM. NM, normal mucosa; OLP, oral lichen planus.

\section{Results}

hMSH2 expression. As shown in Table I, the positive rate of hMSH2 expression in the OLP tissues was $52.94 \%$, which was significantly lower compared with that in the control group $\left(80 \% ; \chi^{2}=7.1993 ; \mathrm{P}<0.05\right)$. In the NM tissue, hMSH2 expression was primarily observed in the cellular nuclei of the basal and spinous layer (Fig. 1). However, in the OLP tissues, positive staining of hMSH2 was predominantly observed in the cytoplasm (Fig. 2).

Associations between hMSH2 expression and clinicopathological features. In the OLP tissues, the expression of hMSH2 was not found to significantly correlate with the gender or age of the patient, or the type of OLP ( $>>0.05$; Table II).

\section{Discussion}

OLP is a common non-infectious disease of the oral mucosa; however, the pathogenesis remains unknown. Currently, OLP is considered to be a potential malignant lesion, and the majority of studies hypothesize that the condition may develop into OSCC $(19,20)$. However, whether OLP exhibits cancerization remains controversial (21), with certain studies considering OLP to be more similar to other benign oral mucosal lesions, and pose no higher risk of cancerization $(22,23)$. The aim of the present study was to observe the protein expression of hMSH2 in the MMR system of OLP, in order to investigate the role of hMSH2 in the occurrence and development of OLP and assess the cancerization trend of OLP.

The MMR system is a basic biological mechanism for maintaining the high fidelity of DNA replication and the stability of the genetic material by controlling genetic mutations. The system is an important barrier to prevent the onset of tumors. MMR proteins always exists in multimeric complex forms to recognize and specifically repair the mismatched bases during DNA replication, and may subsequently induce the apoptosis of cells with severely damaged DNA (24). The inactivation of MMR genes may present as the activation of oncogenes or the inactivation of tumor suppressor genes caused by microsatellite instability, or directly causing mutations in oncogenes or tumor suppressor genes, thereby inducing carcinogenesis $(25,26)$.

To date, nine human MMR genes have been identified, including hMSH2, hMSH6, hMSH5, hMSH4, hMSH3, hMLH1, hPMS1, hPMS2 and hMLH3 (4). hMSH2 was the first human MMR gene to be isolated, which was isolated from human hereditary nonpolyposis colorectal cancer (HNPCC). A number of studies have confirmed that gene mutations of 
Table II. Association between hMSH2 expression and clinicopathological features of patients with OLP.

\begin{tabular}{|c|c|c|c|c|c|c|}
\hline \multirow[b]{2}{*}{ Parameters } & \multirow[b]{2}{*}{ Cases (n) } & \multicolumn{2}{|c|}{ hMSH2 (n) } & \multirow[b]{2}{*}{ Positive rate (\%) } & \multirow[b]{2}{*}{$\chi^{2}$} & \multirow[b]{2}{*}{ P-value } \\
\hline & & + & - & & & \\
\hline Gender & & & & & 0.04 & \\
\hline Male & 22 & 12 & 10 & 54.55 & & $>0.05$ \\
\hline Female & 29 & 15 & 14 & 51.72 & & \\
\hline Age (years) & & & & & 0.14 & \\
\hline$<50$ & 14 & 8 & 6 & 57.14 & & $>0.05$ \\
\hline$\geq 50$ & 37 & 19 & 18 & 51.35 & & \\
\hline Types & & & & & 0.92 & \\
\hline Reticular & 24 & 11 & 13 & 45.83 & & $>0.05$ \\
\hline Erosive/Atrophic & 27 & 16 & 11 & 59.26 & & \\
\hline
\end{tabular}

OLP, oral lichen planus.
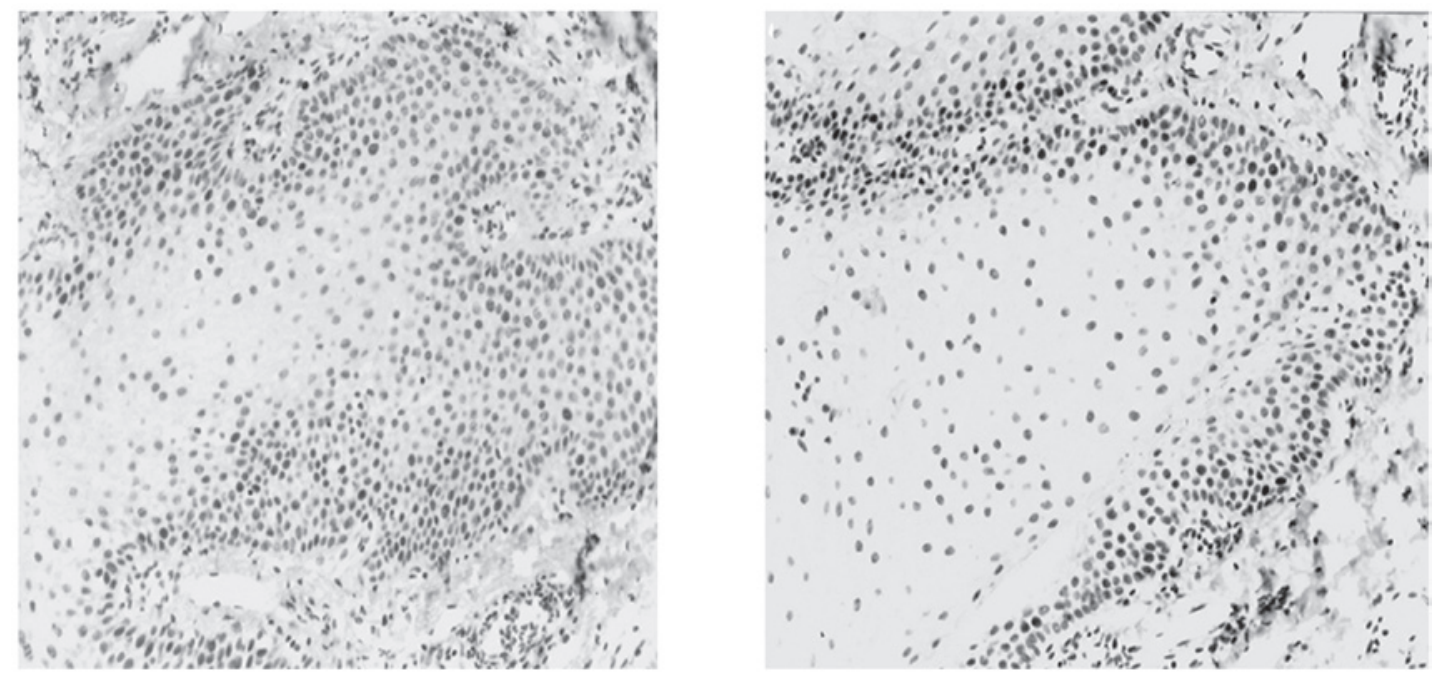

Figure 1. Expression of hMSH2 in normal mucosa (SP; magnification, $\mathrm{x} 400$ ).
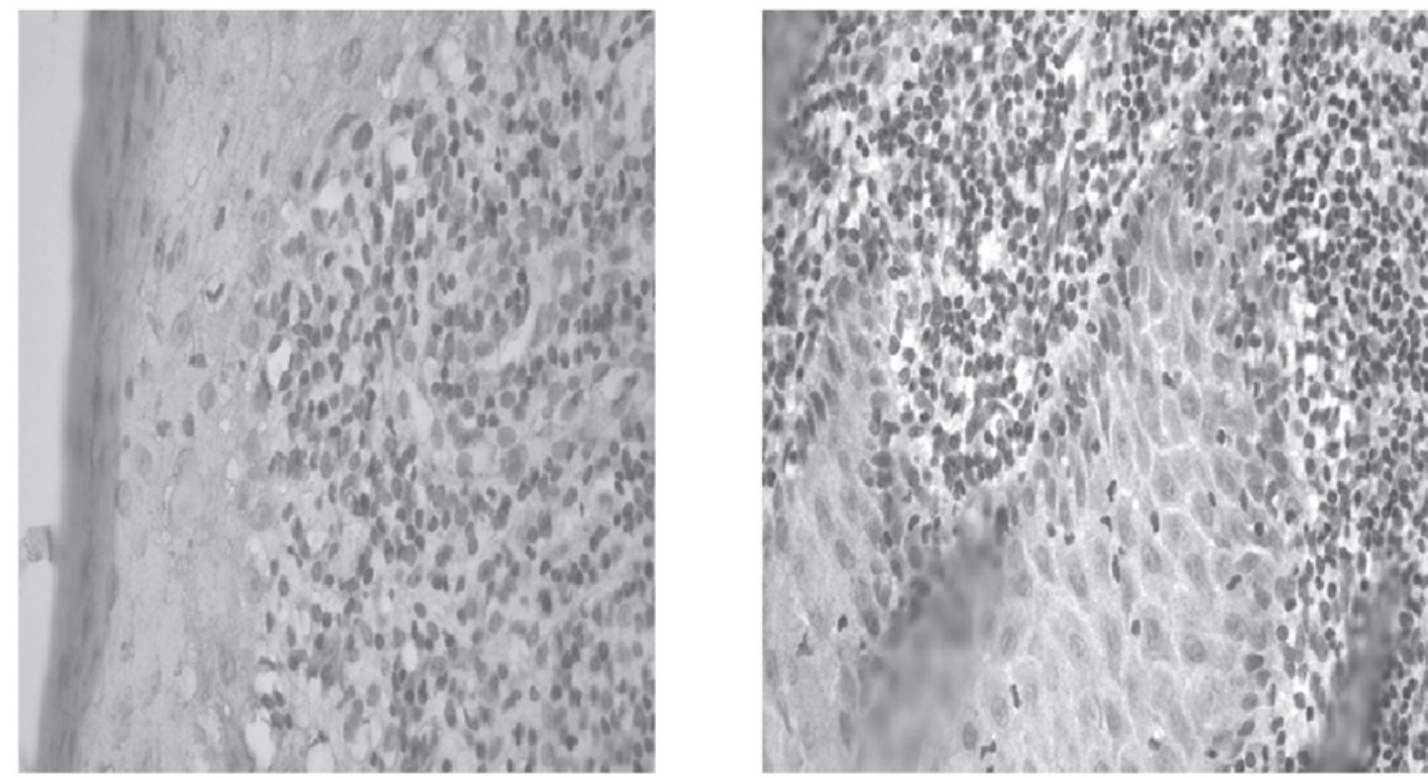

Figure 2. Expression of hMSH2 in patients with oral lichen planus (SP; magnification, $\mathrm{x} 400$ ). 
hMSH2 play crucial roles not only in the pathogenesis of HNPCC (27), but also in the pathogenesis of a variety of other tumors (28-31), including cancer of the endometrium, rectal cancer, ovarian cancer, bladder cancer, as well as OSCC (18). Generally, stronger expression of the MMR proteins can lead to a stronger repairing ability. Thus, the positive rate of hMSH2 in non-tumor tissues is significantly higher compared with that in tumor tissues. For example, the expression of hMSH2 protein in HNPCC, cervical squamous cell carcinoma and prostate cancer is significantly lower compared with that in normal control tissues (32). Lo Muzio et al (18) analyzed the expression of hMSH2 in OSCC and normal oral mucosal tissues using immunohistochemical methods, and found that the deletion of hMSH2 protein may be a potential indicator for OSCC.

In the present study, the positive rate of hMSH2 expression in the OLP tissue was found to be significantly lower compared with that in the control group. Thus, it was hypothesized that defects of MMR function may play certain roles in the occurrence and development of OLP, supporting the hypothesis that OLP is a potentially malignant lesion that poses a cancer risk. Pimenta et al (33) also examined the expression of hMSH2 protein in 26 cases of OLP ( 12 cases of reticular type and 14 cases of atrophic or erosive type) using immunohistochemistry. The authors found that the positive rate of hMSH2 expression was $46.54 \%$ in reticular type OLP, $48.79 \%$ in atrophic or erosive type OLP and $61.29 \%$ in the normal mucosa, which is consistent with the observations of the present study.

In conclusion, the present study demonstrated that hMSH2 expression was significantly reduced in OLP tissues, resulting in MMR defects in the cells, which may subsequently cause genetic instability and lead to the onset of OSCC. However, the exact mechanisms of hMSH2 in the development and carcinogenesis of OLP should be confirmed by further studies.

\section{References}

1. Reenan RA and Kolodner RD: Isolation and characterization of two Saccharomyces cerevisiae genes encoding homologs of the bacterial HexA and Muts mismatch repair proteins. Genetics 132: 963-973, 1992

2. Fishel R, Lescoe MK, Rao MR, et al: The human mutator gene homolog MSH2 and its association with hereditary nonpolyposis colon cancer. Cell 77: 166, 1994.

3. Kunkel TA and Erie DA: DNA mismatch repair. Annu Rev Biochem 74: 681-710, 2005.

4. Manolagas SC and Jilka RL: Bone marrow, cytokines, and bone remodeling. Emerging insights into the pathophysiology of osteoporosis. N Engl J Med 332: 305-311, 1995.

5. Wagner A, Barrows A, Wijnen JT, et al: Molecular analysis of hereditary nonpolyposis colorectal cancer in the United States: high mutation detection rate among clinically selected families and characterization of an American founder genomic deletion of the MSH2 gene. Am J Hum Genet 72: 1088-1100, 2003.

6. Lipkin SM, Wang V, Jacoby R, et al: MLH3: a DNA mismatch repair gene associated with mammalian microsatellite instability. Nat Genet 24: 27-35, 2000.

7. Hsu HS, Wen CK, Tang YA, Lin RK, Li WY, Hsu WH and Wang YC: Promoter hypermethylation is the predominant mechanism in hMLH1 and hMSH2 deregulation and is a poor prognostic factor in nonsmoking lung cancer. Clin Cancer Res 11: 5410-5416, 2005.

8. Salonen L, Axéll T and Helldén L: Occurrence of oral mucosal lesions, the influence of tobacco habits and an estimate of treatment time in an adult Swedish population. J Oral Pathol Med 19: $170-176,1990$

9. Carrozzo M: How common is oral lichen planus? Evid Based Dent 9: 112-113, 2008
10. Dissemond J: Oral lichen planus: an overview. J Dermatolog Treat 15: 136-140, 2004.

11. Carrozzo M and Pellicano R: Lichen planus and hepatitis $C$ virus infection: an updated critical review. Minerva Gastroenterol Dietol 54: 65-74, 2008.

12. Lanfranchi-Tizeira HE, Aguas SC and Sano SM: Malignant transformation of atypical oral lichen planus: a review of 32 cases. Med Oral 8: 2-9, 2002 (In English and Spanish).

13. Warnakulasuriya S, Johnson NW and van der Waal I: Nomenclature and classification of potentially malignant disorders of the oral mucosa. J Oral Pathol Med 36: 575-580, 2007.

14. Bombeccari GP, Guzzi G, Tettamanti M, Giannì AB, Baj A, Pallotti F and Spadari F: Oral lichen planus and malignant transformation: a longitudinal cohort study. Oral Surg Oral Med Oral Pathol Oral Radiol Endod 112: 328-334, 2011.

15. Georgakopoulou EA, Achtari MD, Achtaris M, Foukas PG and Kotsinas A: Oral lichen planus as a preneoplastic inflammatory model. J Biomed Biotechnol 2012: 759626, 2012.

16. National Centre for Biotechnology Information. http://www.ncbi. nlm.nih.gov/SNP. Accessed October 16, 2013.

17. UniProt Knowledgebase: UniProtKB/Swiss-Prot. http://www. expasy.ch/sprot/sprot-top.html. Accessed October 16, 2013.

18. Lo Muzio L, Nocini P, Mignogna MD, et al: Immunocytochemical detection of hMSH2 and hMLH1 expression in oral SCC. Anticancer Res 19: 933-940, 1999.

19. van der Meij EH, Schepman KP, Smeele LE, van der Wal JE, Bezemer PD and van der Waal I: A review of the recent literature regarding malignant transformation of oral lichen planus. Oral Surg Oral Med Oral Pathol Oral Radiol Endod 88: 307-310, 1999.

20. Scully C, Beyli M, Ferreiro MC, et al: Update on oral lichen planus: etiopathogenesis and management. Crit Rev Oral Biol Med 9: 86-122, 1998.

21. Accurso BT, Warner BM, Knobloch TJ, Weghorst CM, Shumway BS, Allen CM and Kalmar JR: Allelic imbalance in oral lichen planus and assessment of its classification as a premalignant condition. Oral Surg Oral Med Oral Pathol Oral Radiol Endod 112: 359-366, 2011.

22. O'Flatharta C, Leader M, Kay E, Flint SR, Toner M, Robertson W and Mabruk MJ: Telomerase activity detected in oral lichen planus by RNA in situ hybridisation: not a marker for malignant transformation. J Clin Pathol 55: 602-607, 2002.

23. Eisenberg E. Oral lichen planus: a benign lesion. J Oral Maxillofac Surg 58: 1278-1285, 2000.

24. Lynch HT, Lynch JF, Lynch PM and Attard T: Hereditary colorectal cancer syndromes: molecular genetics, genetic counseling, diagnosis and management. Fam Cancer 7: 27-39, 2008.

25. Amira AT, Mouna T, Ahlem B, et al: Immunohistochemical expression pattern of MMR protein can specifically identify patients with colorectal cancer microsatellite instability. Tumor Biol 35: 6283-6291, 2014.

26. Bairwa NK, Saha A, Gochhait S, Pal R, Gupta V and Bamezai RN: Microsatellite instability: an indirect assay to detect defects in the cellular mismatch repair machinery. Methods Mol Biol 1105: 497-509, 2014.

27. Zhang R, Qin W, Xu GL, Zeng FF and Li CX: A meta-analysis of the prevalence of somatic mutations in the hMLH1 and hMSH2 genes in colorectal cancer. Colorectal Dis 14: e80-e89, 2012.

28. González L, Ortiz AP, Suárez EL, et al: Case-case study of factors associated to hMLH1, hMSH2, and hMSH6 protein expression among endometrial cancer patients of the University District Hospital of San Juan, Puerto Rico. Int J Gynecol Cancer 22: 826-829, 2012.

29. Lu FI, Gilks CB, Mulligan AM, et al: Prevalence of loss of expression of DNA mismatch repair proteins in primary epithelial ovarian tumors. Int J Gynecol Pathol 31: 524-531, 2012.

30. Vageli DP, Giannopoulos S, Doukas SG, et al: Mismatch repair hMSH2, hMLH1, hMSH6 and hPMS2 mRNA expression profiles in precancerous and cancerous urothelium. Oncol Lett 5: 283-294, 2013.

31. Gu MJ, Bae YK, Kim A, et al; Korean Small Intestinal Cancer Study Group: Expression of hMLH1, hMSH2 and hMSH6 in small intestinal carcinomas. Hepatogastroenterology 59: 2228-2232, 2012.

32. Ciavattini A, Piccioni M, Tranquilli AL, Filosa A, Pieramici T and Goteri G: Immunohistochemical expression of DNA mismatch repair (MMR) system proteins (hMLH1, hMSH2) in cervical preinvasive and invasive lesions. Pathol Res Pract 201: 21-25, 2005.

33. Pimenta FJGS, Pinheiro MDGR and Gomez RS: Expression of $\mathrm{hMSH} 2$ protein of the human DNA mismatch repair system in oral lichen planus. Int J Med Sci 1: 146-151, 2004. 